\title{
Independent pair parton interactions-model of hadron interactions
}

\author{
I.M. Dremin $*$ and V.A. Nechitaild \\ Lebedev Physical Institute, Moscow
}

(Dated: November 25, 2018)

\begin{abstract}
A model of independent pair parton interactions is proposed, according to which, hadron interactions are represented by a set of independent binary parton collisions. The final multiplicity distribution is described by a convolution of the negative binomial distributions in each of the partonic collisions. As a result, it is given by a weighted sum of negative binomial distributions with parameters multiplied by the number of active pairs. Its shape and moments are considered. Experimental data on multiplicity distributions in high energy $p \bar{p}$ processes are well fitted by these distributions. Predictions for the CERN Large Hadron Collider and higher energies are presented. The difference between $e^{+} e^{-}$and $p \bar{p}$ processes is discussed.
\end{abstract}

\section{INTRODUCTION}

Hadron interactions used to be considered as proceeding via collisions of their constituent partons. In preparton times, their role was played by pions, and the onemeson exchange model [1] dominated. Pions were treated as hadron constituents. Their high energy interaction produced a ladder of one-pion $t$-channel exchanges with blobs of low energy pion-pion interactions. This is the content of the multiperipheral model. These blobs were first interpreted as $\rho$ mesons 2] and later called fireballs [3], clusters 4] or clans [5] when higher mass objects were considered. Multiperipheral dynamics tells us that the number of these blobs is distributed according to the Poisson law. It was argued that its convolution with the distribution of the number of pions produced in each center can lead to a negative binomial distribution (NBD) of created particles first introduced in [6]. This supposition fits experimental data on multiplicity distributions of $p p$ reactions at tens of $\mathrm{GeV}$ quite well. However, at higher energies this fit by a single NBD becomes unsatisfactory. A shoulder appears at high multiplicities. It is quite natural to ascribe it to multiple parton-parton collisions 17, 8, 9, 10], which could lead, e.g., to two-, three-, and so on, ladder formation [11, 12, 13], and/or to different (soft, hard) types of interactions [14, 15]. They become increasingly important as the collision energy is increased. Better fits are achieved at the expense of a larger number of adjustable parameters.

This shortcoming can be minimized if one assumes that each of the high energy binary parton collisions is independent of others proceeding simultaneously. With this supposition, the whole process is described as a set of independent pair parton interactions (the IPPI model). In fact, we assume democracy in sharing the initial energy of colliding hadrons among their constituents. The effective multiplicity of particles produced by a pair of initial partons does not depend on how many other pairs interact or on what these interacting partons are (quarks

*Electronic address: dremin@lpi.ru

${ }^{\dagger}$ Electronic address: nechit@lpi.ru or gluons). While parton energies vary widely at a given hadron energy, the mean amount of energy involved in each parton-parton collision can be comparable. Therefore, we hope that this simplification is valid at very high energies for such global characteristics as multiplicity distributions which result from an average over the whole phase space. If necessary, this supposition can be relaxed by introducing the parton distribution functions (PDF). The inclusive distributions would call for a more detailed description. The IPPI model does not imply that there are no correlations between particles. They are intrinsic in each binary collision and in their convolution. Surely, further correlations between these interacting pairs of partons, of both dynamical and kinematical origin, can be introduced. Nevertheless, the simplest model with minimum parameters and its most general characteristics such as multiplicity distributions should be treated first.

\section{IPPI MODEL AND MULTIPLICITY DISTRIBUTIONS}

For multiplicity distributions, we suppose that a parton-parton collision gives rise to a negative binomial distribution of its products with parameters independent of the colliding partons because the energy is equally shared between them. Therefore, the resulting distribution of independent pair parton interactions is given by a sum of convolutions of processes with different number of participating pairs of partons weighted according to their probabilities. Thus one can write

$$
\begin{aligned}
& P(n ; m, k)=\sum_{j=1}^{j_{\max }} w_{j} P_{j}(n ; m, k) \\
= & \sum_{j=1}^{j_{\max }} w_{j} \sum_{\left(n_{p}\right)} \prod_{p=1}^{j} P_{N B D}\left(n_{p} ; m, k\right) .
\end{aligned}
$$

Here, $P(n ; m, k)$ is the probability of creating $n$ particles, which depends on the parameters of the NBD distribution $m$ and $k, n_{p}$ is the number of particles produced by the $p$ th pair, $w_{j}$ is the probability for the $j$ th pair to be 
active and $j_{\max }$ is a number of active pairs. Therefore, the following equations are valid

$$
\sum_{p=1}^{j} n_{p}=n, \quad \sum_{j=1}^{j_{\max }} w_{j}=1 .
$$

The symbolical notation $\sum_{\left(n_{p}\right)}$ means the convolution of NBD expressions subject to the first equation in (2), i.e., the sum must be taken only over those parton collisions whose multiplicities $n_{p}$ sum up to the total number of produced particles $n$. The NBD shape

$P_{N B D}\left(n_{p} ; m, k\right)=\frac{\Gamma\left(n_{p}+k\right)}{\Gamma\left(n_{p}+1\right) \Gamma(k)}\left(\frac{m}{k}\right)^{n_{p}}\left(1+\frac{m}{k}\right)^{-n_{p}-k}$ is characterized by two parameters $m$ and $k$, corresponding to the mean multiplicity and the dispersion $D_{1}$ of the distribution for a single interaction,

$$
k^{-1}=\left(D_{1}^{2}-m\right) / m^{2} .
$$

It is our supposition that, weighted by the parton distribution functions, such an interaction at a fixed partonparton energy leads to a NBD. This is based on the success of low energy fits. In multiperipheral-type models with a Poisson distribution of created blobs, this would imply a gamma distribution of the decay products of these blobs.

For example, the formula (11) explicitly written for three active parton pairs is as follows

$$
\begin{array}{r}
P(n ; m, k)=\frac{1}{(1+k / m)^{n}(1+m / k)^{k} \Gamma(k)}\left(w_{1} \frac{\Gamma(n+k)}{\Gamma(n+1)}+w_{2} \frac{1}{\Gamma(k)(1+m / k)^{k}} \sum_{n_{1}=0}^{n} \frac{\Gamma\left(n_{1}+k\right) \Gamma\left(n-n_{1}+k\right)}{\Gamma\left(n_{1}+1\right) \Gamma\left(n-n_{1}+1\right)}\right. \\
\left.+w_{3} \frac{1}{\Gamma^{2}(k)(1+m / k)^{2 k}} \sum_{n_{1}=0}^{n} \frac{\Gamma\left(n_{1}+k\right)}{\Gamma\left(n_{1}+1\right)} \sum_{n_{2}=0}^{n-n_{1}} \frac{\Gamma\left(n_{2}+k\right) \Gamma\left(n-n_{1}-n_{2}+k\right)}{\Gamma\left(n_{2}+1\right) \Gamma\left(n-n_{1}-n_{2}+1\right)}+\cdots\right)
\end{array}
$$

Each of the three terms in this sum represents a negative binomial distribution because

$$
\sum_{n_{1}=0}^{n} \frac{\Gamma\left(n_{1}+k\right) \Gamma\left(n-n_{1}+l k\right)}{\Gamma\left(n_{1}+1\right) \Gamma\left(n-n_{1}+1\right)}=\frac{\Gamma(k) \Gamma(l k) \Gamma(n+(l+1) k)}{\Gamma((l+1) k) \Gamma(n+1)} .
$$

This is a general remarkable property of negative binomial distributions: their convolutions result again in NBD functions with parameters multiplied by the number of convolutions. Thus Eq. (1) can be rewritten as follows

$$
P(n ; m, k)=\sum_{j=1}^{j_{\max }} w_{j} P_{N B D}(n ; j m, j k) .
$$

This is the main equation of the IPPI model. One gets a sum of negative binomial distributions with shifted maxima and larger widths for a larger number of collisions. No new adjustable parameters appear in the distribution for $j$ pairs of colliding partons. All parameters are expressed in terms of the products of parameters for a single collision and the number of collisions. Both the mean multiplicity and dispersion $D_{j}^{2}$ for the process with $j$ active parton pairs are proportional to $j$. In the total multiplicity distribution, the distributions for collisions of $j$ pairs of partons, $P_{N B D}$, are just weighted with their probabilities $w_{j}$, which are determined by collision dynamics and, in principle, can be evaluated if some model is adopted (e.g., see [11, 12, 13]).

An increase in the number of interacting pairs of partons in the IPPI model with energy gives rise to more probabilities $w_{j}$ different from zero. Certainly, all the parameters $w_{j}, m, k$ depend on energy. This dependence is implied but not shown explicitly in the above formulas. One can hope that at asymptotically high energies the probability for $j$ pairs of independent interactions $w_{j}$ is the product of $j$ probabilities $w_{1}$ for one pair,

$$
w_{j}=w_{1}^{j} .
$$

From the normalization condition

$$
\sum_{j=1}^{j_{\max }} w_{j}=\sum_{j=1}^{j_{\max }} w_{1}^{j}=1,
$$

one can find $w_{1}$ if $j_{\max }$, which is determined by the maximum number of parton interactions at a given energy, is known. In fact, the value of $w_{1}$ ranges between 1 at low energies (for $j_{\max }=1$ ) and 0.5 at asymptotics where $j_{\max }$ tends to infinity. With energy increase, it approaches the second value from above, passing through some thresholds, and is already quite close to it at the present highest energies.

Thus we are left with only two parameters of the model, $m$ and $k$, which can be found from fits of experimental data. The dependence on the number of collisions $j_{\max }$ and on the probabilities $w_{j}$ is determined by the behavior of the moments of probabilities

$$
M_{r}=\sum_{j=1}^{j_{\max }} w_{j} j^{r}
$$


as explicitly shown in the Appendix for ranks $r \leq 5$. In particular, the average multiplicity is given by

$$
\langle n\rangle=m M_{1}
$$

If one assumes some extrapolation of $\langle n\rangle$ to higher energies, it can be used for prediction of the distributions. Let us emphasize that the asymptotic behavior of $m$ is directly related to that of the mean multiplicity,

$$
\left\langle n_{a s}\right\rangle=m_{a s} \sum_{j=1}^{\infty} j w_{1}^{j}=m_{a s} \frac{w_{1}}{\left(1-w_{1}\right)^{2}}=2 m_{a s} .
$$

The value of $m$ is usually quite close to the position of the maximum of the distribution. Thus the relation (12) tells us that in the IPPI model the asymptotic mean multiplicity is about twice larger than the location of its maximum determined mainly by a single parton-parton interaction. One can expect that the asymptotic relation for the probabilities (8) becomes valid only at energies where four or more pairs are already active. This series becomes a polynomial at finite energies. In practice, the threshold effects should also be taken into account at finite energy. They would somewhat suppress $w_{j}$ at the largest $j$ and, correspondingly, enlarge the role of oneand two-pair interactions.

In 13], the energy dependence of the probabilities $w_{j}$ was estimated according to the multiladder exchange model 12 but Poisson distributions were used for each of the ladders. The probabilities are given by the following normalized expressions

$w_{j}\left(\xi_{j}\right)=\frac{p_{j}}{\sum_{j=1}^{j_{\max }} p_{j}}=\frac{1}{j Z_{j}\left(\sum_{j=1}^{j_{\max }} p_{j}\right)}\left(1-e^{-Z_{j}} \sum_{i=0}^{j-1} \frac{Z_{j}^{i}}{i !}\right)$

where

$$
\xi_{j}=\ln \left(s / s_{0} j^{2}\right), \quad Z_{j}=\frac{2 C \gamma}{R^{2}+\alpha_{P}^{\prime} \xi_{j}}\left(\frac{s}{s_{0} j^{2}}\right)^{\Delta}
$$

with numerical parameters obtained from fits of the experimental data on total and elastic scattering cross sections: $\gamma=3.64 \mathrm{GeV}^{-2}, \quad R^{2}=3.56 \mathrm{GeV}^{-2}, \quad C=1.5$, $\Delta=\alpha_{P}-1=0.08, \quad \alpha_{P}^{\prime}=0.25 \mathrm{GeV}^{-2}, s_{0}=1 \mathrm{GeV}^{2}$.

Below, we will use both possibilities (8) and (13) in our attempts to describe the experimental data. The probabilities $w_{j}$ are different for each (see Table \). In the IPPI model they decrease exponentially with increasing number of active partons, while in the ladder model they are inversely proportional to this number with additional suppression at large $j$ due to the term in parentheses in (13). This is the result of the modified eikonal approximation.

We show the values $w_{j}$ for $3-6$ pairs calculated according to Eq. (8) in the left-hand side of Table \and according to Eq. (13) in its right-hand side. These values $j_{\max }$ are chosen because they will be used in the comparison with experiment. In particular, we shall choose $j_{\max }=3$
TABLE I: The values of $w_{j}$ according to Eq. (8) (left-hand side) and Eq. (13) (right-hand side).

\begin{tabular}{|c||c|c|c|c||c|c|c|c|}
\hline$j_{\max }$ & 3 & 4 & 5 & 6 & 3 & 4 & 5 & 6 \\
\hline$w_{1}$ & 0.544 & 0.519 & 0.509 & 0.504 & 0.562 & 0.501 & 0.450 & 0.410 \\
$w_{2}$ & 0.295 & 0.269 & 0.259 & 0.254 & 0.278 & 0.255 & 0.236 & 0.219 \\
$w_{3}$ & 0.161 & 0.140 & 0.131 & 0.128 & 0.160 & 0.153 & 0.152 & 0.147 \\
$w_{4}$ & 0 & 0.072 & 0.067 & 0.065 & 0 & 0.091 & 0.100 & 0.104 \\
$w_{5}$ & 0 & 0 & 0.034 & 0.033 & 0 & 0 & 0.062 & 0.073 \\
$w_{6}$ & 0 & 0 & 0 & 0.016 & 0 & 0 & 0 & 0.047 \\
\hline
\end{tabular}

at 300 and $546 \mathrm{GeV}, 4$ at 1000 and $1800 \mathrm{GeV}, 5$ at 14 $\mathrm{TeV}$, and 6 at $100 \mathrm{TeV}$ (see below).

One can clearly see the difference between the two approaches. The value of $w_{1}$ is always larger than 0.5 in the IPPI model while it can become less than 0.5 in the ladder model [11, 12] at high energies. In the ladder model, $w_{j}$ depend explicitly on energy (not only on the $j_{\max }$ cutoff). We show their values at 546 and $1800 \mathrm{GeV}$ in the right-hand side columns of $j_{\max }=3$ and 4 . Those at 300 and $1000 \mathrm{GeV}$ are larger for $w_{1}$ by about $1 \%$ and smaller for $w_{3}$ by about $3 \%$. When the energy increases, the processes with a larger number of active pairs play a more important role in the ladder approach compared to the IPPI model. Thus, the $j_{\max }$ cutoff is also more essential there.

In principle, one can immediately try a two-parameter fit of experimental multiplicity distributions using Eq. (7) if $w_{j}$ are known. However, the use of their moments is preferred as shown below.

\section{MOMENTS OF MULTIPLICITY DISTRIBUTIONS}

The shapes of the multiplicity distributions $P(n)$ usually look quite complicated. Often, they are better represented by their moments, which also contain complete information. The easiest way to define them is to introduce the generating function

$$
G(z)=\sum_{n=0}^{\infty} P(n)(1+z)^{n} .
$$

In what follows, we will use the so-called unnormalized factorial $\mathcal{F}_{q}$ and cumulant $\mathcal{K}_{q}$ moments defined according to the formulas

$$
\begin{gathered}
\mathcal{F}_{q}=\sum_{n} P(n) n(n-1) \ldots(n-q+1)=\left.\frac{d^{q} G(z)}{d z^{q}}\right|_{z=0}, \\
\mathcal{K}_{q}=\left.\frac{d^{q} \ln G(z)}{d z^{q}}\right|_{z=0} .
\end{gathered}
$$

They correspondingly determine the total and genuine correlations among the particles produced (for more details, see [16]). For $q=1$, they define the mean multiplicity; the second moment is related to the width of 
the distribution, etc. The factorial moments are evaluated from experimental data according to their definition (16). Both $\mathcal{F}_{q}$ and $\mathcal{K}_{q}$ grow, however, extremely fast with their ranks. Therefore, it is more convenient to use [17] their ratio $H_{q}=\mathcal{K}_{q} / \mathcal{F}_{q}$, where these dependencies partly cancel. This ratio is easy to find from iterative formulas:

$$
H_{q}=1-\sum_{p=1}^{q-1} \frac{\Gamma(q)}{\Gamma(p+1) \Gamma(q-p)} H_{q-p} \frac{\mathcal{F}_{p} \mathcal{F}_{q-p}}{\mathcal{F}_{q}}
$$

once the factorial moments have been evaluated. Thus, both $\mathcal{F}_{q}$ and $H_{q}$ are determined by experimental data according to Eqs. (16) and (18).

Recall that these ratios appear quite naturally in QCD as the solutions of the equations for the generating functions of multiplicity distributions [17]. Therefore, their use is especially informative because one can compare experimental results and model calculations with analytical QCD predictions for jets in $e^{+} e^{-}$annihilation as reviewed in [18]. QCD predicts a very specific behavior of the $H_{q}$ moments as functions of ranks $q$ and energy. It has been shown [17, 19] that $H_{q}$ for jets at the present energies (SLC, LEP) should oscillate, and this prediction has been confirmed by experimental results [20, 21, 22]. The first minimum is located near $q=5$ at $\mathrm{Z}^{0}$ energy. At higher energies, this minimum moves to larger values of $q$; the oscillations become less pronounced and disappear in the asymptotics where $H_{\alpha}=1 / q^{2}$. Moreover, these oscillations have been found [18, 20] even for experimentally studied $p p$ and $A A$ collisions. In these cases, they can be ascribed to the multicomponent structure of the processes. Such a structure is incorporated in the IPPI model according to Eq. (1). We will see if it is enough to describe experimental data.

Let us emphasize that $H_{q}$ moments are very sensitive to minute details of the multiplicity distributions and can be used to distinguish between different models and experimental data. However, one should be warned that the amplitudes of the oscillations strongly depend on the multiplicity distribution cutoff due to limited experimental statistics (or by another reasoning) if the experiment is done at rather low multiplicities. There are no cutoffs in analytical expressions for $H_{q}$. One can control the influence of cutoffs by shifting them appropriately. The qualitative features persist nevertheless. In what follows, we consider very high energy processes where the cutoff due to experimental statistics is practically insignificant.

The IPPI model predicts new special features of the moments $\mathcal{F}_{q}$ and $H_{q}$. The factorial moments of the distribution (7) are

$$
\mathcal{F}_{q}=\sum_{j=1}^{j_{\max }} w_{j} \frac{\Gamma(j k+q)}{\Gamma(j k)}\left(\frac{m}{k}\right)^{q}=f_{q}(k)\left(\frac{m}{k}\right)^{q}
$$

with

$$
f_{q}(k)=\sum_{j=1}^{j_{\max }} w_{j} \frac{\Gamma(j k+q)}{\Gamma(j k)}=k \sum_{j=1}^{j_{\max }} w_{j} j(j k+1) \cdots(j k+q-1) \text {. }
$$

The cumulant moments are written as

$$
\mathcal{K}_{q}=\kappa_{q}(k)\left(\frac{m}{k}\right)^{q}
$$

The explicit $k$ dependence of $f_{q}(k)$ and $\kappa_{q}(k)$ for $q \leq 5$ is shown in the Appendix. For $H_{q}$ moments one gets

$$
H_{q}=1-\sum_{p=1}^{q-1} \frac{\Gamma(q)}{\Gamma(p+1) \Gamma(q-p)} H_{q-p} \frac{f_{p} f_{q-p}}{f_{q}} .
$$

Note that according to Eq. (22) $H_{q}$ are functions of the parameter $k$ only and do not depend on $m$ in the IPPI model, because the $m$ dependence of factorial and cumulant moments is the same. This remarkable property of the $H_{q}$ moments provides an opportunity to fit the same results with a smaller number of parameters. If the $w_{j}$ 's are given by Eq. (9), the only adjustable parameter left is $k$. These moments decrease with increase of $k$ and $q$.

Once the parameter $k$ is found from fits of $H_{q}$, it is possible to get another parameter $m$ by rewriting Eq. (19) as follows:

$$
m=k\left(\frac{\mathcal{F}_{q}}{f_{q}(k)}\right)^{1 / q}
$$

This formula is a sensitive test for the whole approach because it states that the definite ratio of $q$-dependent functions to the power $1 / q$ becomes $q$ independent if the model is correct. Moreover, this statement should be valid only for those values of $k$ that are determined from $H_{q}$ fits. Therefore, it can be considered as a criterion for a proper choice of $k$ and for the model validity, in general.

One substitutes the experimentally determined values of factorial moments, divides them by the theoretical functions $f_{q}$ given by Eq. (20), and examines whether this ratio to the power $1 / q$ is independent of $q$ at $k$ values found previously from $H_{q}$ fits. If the answer is positive, the parameter $m$ is known according to Eq. (23). If not, the model should be modified. With parameters $k$ and $m$ found, one can try to fit the shapes of experimentally measured multiplicity distributions directly. This is another test of the self-consistency of the IPPI model.

At the same time, the value of $m$ determines the position of the peak of the multiplicity distribution. For a given $\langle n\rangle$, it can be used to check the choice of probabilities $w_{j}$ according to Eq. (11).

Recall that both parameters $m$ and $k$ depend on the energy of the colliding hadrons $s$. This dependence can be determined from fits of experimentally found values of $H_{q}$ and $\mathcal{F}_{q}$ as explained above. To extrapolate it to higher energies, one should use some guesses. Since $m$ has the meaning of the average multiplicity of a binary parton collision, it should behave similarly to the mean multiplicity of the whole process. The latter is usually fitted by a logarithmic dependence with some log-squared terms added. No experience has been gained yet for the parameter $k$. 
The Poisson distribution possesses the same property of convolutions which made it possible to get Eq. (7) for NBD distributions. Therefore, all the above relations are valid for a model with convoluted Poisson distributions. Actually, they can be obtained in the limit $k \rightarrow \infty$. For example, the factorial moments are $\mathcal{F}_{q}^{(\text {Poisson })}=m^{q} M_{q}$.

\section{COMPARISON WITH EXPERIMENT}

We have compared IPPI model conclusions with experimental multiplicity distributions of the E735 Collaboration [23] for $p \bar{p}$ collisions at energies 300, 546, 1000, and $1800 \mathrm{GeV}$ extrapolated [10, 24] to the full phase space. The multiplicity of charged particles was divided by 2 to get the multiplicity of particles with the same charge. Then the above formulas for the moments were used. Correspondingly, the parameters $m$ and $k$ refer to these distributions.

An analysis of experimental data done in 10 has shown that two parton pairs are already active at energies above $120 \mathrm{GeV}$. The thresholds for triple or more parton-parton collisions are less definite. They depend on the form of the multiplicity distribution adopted for a single collision. We assume that three parton pairs are active at 300 and $546 \mathrm{GeV}$ and four at 1000 and 1800 $\mathrm{GeV}$ with NBDs for a single collision. We use these values in our calculations.

Factorial and $H_{q}$ moments were obtained from experimental data on $P(n)$ according to Eqs. (16) and (18). Experimental $H_{q}$ moments were fitted by Eq. (22) to get the parameters $k(E)$ of the IPPI model. We show in Fig. 1 how perfect are these fits at $1.8 \mathrm{TeV}$ for $k$ equal to 3.7 (solid line) and 4.4 (dash-dotted line). At this energy, we consider four active parton pairs with $w_{j}$ given by Eq. (9) (the second column in Table I). It is surprising that the oscillations of $H_{q}$ moments are so well reproduced with one adjustable parameter $k$. The general tendency of this quite complicated oscillatory dependence is clearly seen.

With these values of the parameter $k$, we have checked whether $m$ is constant as a function of $q$. Experimental factorial moments and IPPI values for $f_{q}$ were inserted in Eq. (23). The $m(q)$ dependence is shown in Fig. 2 for the same values of $k=4.4$ (squares) and 3.7 (circles) and for a much larger value 7.5 (triangles). The constancy of $m$ is satisfied with an accuracy better than $1.5 \%$ for $k=4.4$ up to $q=16$. The upper and lower lines in Fig. 2 demonstrate clearly that this condition substantially bounds the admissible variations of $k$.

It is well known that experimental cutoffs of multiplicity distributions due to the limited statistics of an experiment can influence the behavior of $H_{q}$ moments. Consequently, they impose some limits on the $q$ values that can be considered when a comparison is done. Higher rank moments can be evaluated if larger multiplicities have been measured. To estimate the admissible range of $q$, we use the results obtained in QCD. Characteristic mul-

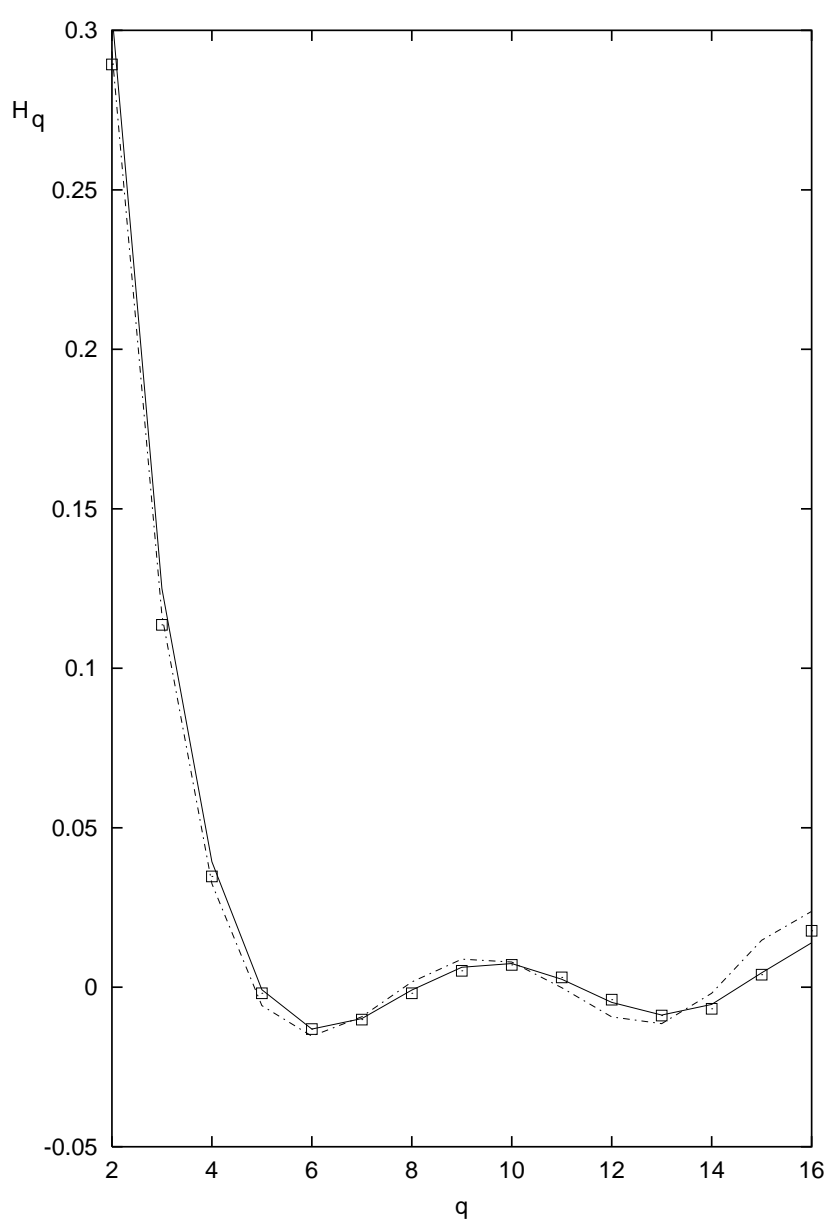

FIG. 1: A comparison of $H_{q}$ moments derived from experimental data at $1.8 \mathrm{TeV}$ (squares) with their values calculated with parameter $k=4.4$ (dash-dotted line) and 3.7 (solid line).

tiplicities that determine the moment of the rank $q$ can be found. By inverting this relation, one can write the asymptotic expression for the characteristic range of $q$ [25]. This provides the bound $q_{\max } \approx C n_{\max } /\langle n\rangle$ where $C \approx 2.5527$. However, it underestimates the factorial moments. Moreover, the first moment is not properly normalized (it becomes equal to $2 / C$ instead of 1 ). The strongly overestimated values (however, with a correct normalization of the first moment) are obtained if $C$ is replaced by 2 . Hence, one can say that the limiting values of $q$ are given by the inequalities

$$
2 n_{\max } /\langle n\rangle\left\langle q_{\max } \leq C n_{\max } /\langle n\rangle .\right.
$$

The ratio $n_{\max } /\langle n\rangle$ measured by the E735 Collaboration at $1.8 \mathrm{TeV}$ is about 5. Thus, $q_{\max }$ should be in the interval between 10 and 13 . The approximate constancy of $m$ and proper fits of $H_{q}$ demonstrated above persist to even higher ranks.

The same-charge multiplicity distribution at $1.8 \mathrm{TeV}$ has been fitted with the parameters $m=12.94$ and $k=$ 4.4 as shown in Fig. 3 (solid line). To estimate the accuracy of the fit, we calculated $\sum_{n=1}^{125}\left[P_{t}(n)-P_{e}(n)\right]^{2} / \Delta^{2}$ 


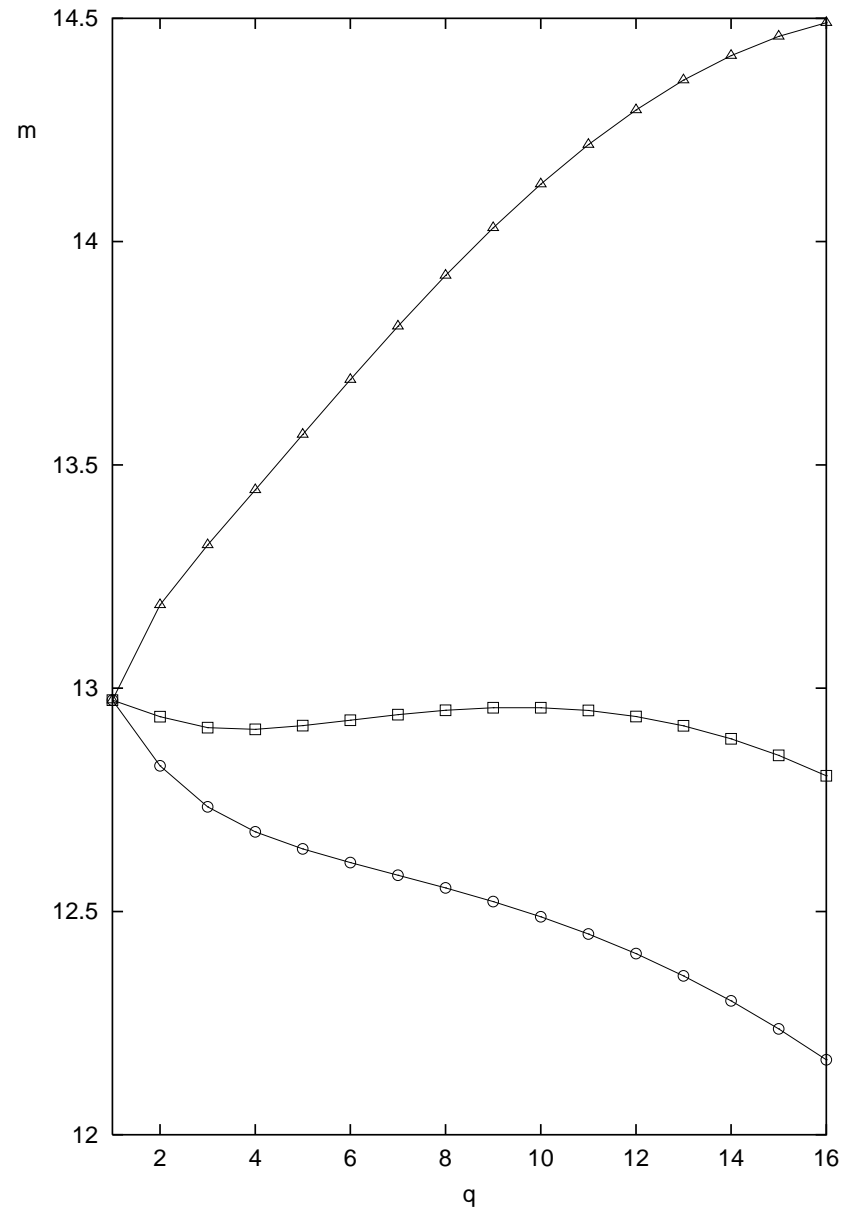

FIG. 2: $\quad$ The $q$ dependence of $m$ for $k=4.4$ (squares), 3.7 (circles), and 7.5 (triangles).

over all 125 experimental points. Here, $P_{t}, P_{e}$ are the theoretical and experimental distributions and $\Delta$ is the total experimental error. It includes both statistical and systematical errors. Note that the latter are large at low multiplicities in the E735 data. This sum is equal to 50 for 125 degrees of freedom. No minimization of it was attempted. This is twice better than the three-parameter fit by a generalized NBD considered in 26]. A Poisson distribution of particles in binary collisions is completely excluded. This is shown in Fig. 3 by the dash-dotted line.

We show in Fig. 4 the decomposition of the fit in Fig. 3 to processes with different numbers $j$ of parton pairs involved in collision. It is seen that the locations of their maxima are approximately proportional to $j$.

The same procedure has been applied to data at energies 300, 546, and $1000 \mathrm{GeV}$. As stated above, we have assumed that three binary parton collisions are active at 300 and $546 \mathrm{GeV}$ and four at $1000 \mathrm{GeV}$. We plot in Figs. 5 and 6 the energy dependence of the parameters $m$ and $k$. The parameter $m$ increases logarithmically with energy. This is expected [see [11)] because increase of $M_{1}$ due to increasing numbers of active pairs at these ener-

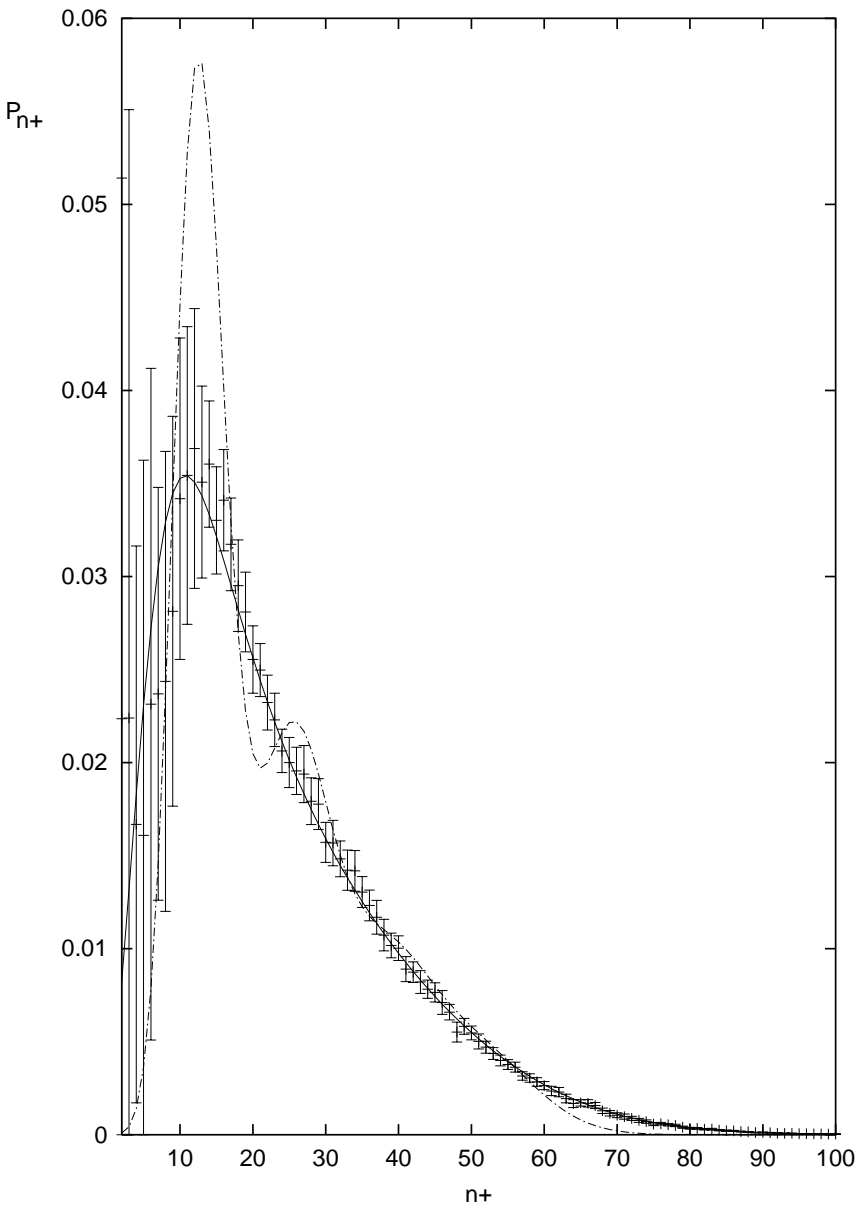

FIG. 3: The multiplicity distribution at $1.8 \mathrm{TeV}$ and its fit at $m=12.94, k=4.4$ (solid line). The dash-dotted line demonstrates what would happen if the NBD were replaced by Poisson distribution.

gies leads to a somewhat faster than logarithmic increase of the average multiplicity in accordance with experimental observations. The energy dependence of $k$ (crosses) is more complicated and rather irregular.

We tried to ascribe the latter to the fact that the effective values of $k$, which we actually find from these fits, depend on the effective number of parton interactions, i.e., on the $w_{j}$ variation at a threshold. The threshold effects can be important in this energy region. Then the simple relation (8) is invalid. This influences the functions $f_{q}(k)$ [Eq. (20)] and, consequently, $H_{q}$ calculated from Eq. (22). One can reduce the effective number of active pairs to about 2.5 at $300 \mathrm{GeV}$ and 3.5 at $1000 \mathrm{GeV}$ if one chooses the following values of $w_{j}: 0.59,0.34$, and 0.07 at $300 \mathrm{GeV}$ and $0.54,0.29,0.14$, and 0.03 at 1000 $\mathrm{TeV}$ instead of those calculated according to Eq. (8) and shown in Table【 This gives rise to values of $k$ which are not drastically different from the previous ones. However, the quality of the fits becomes worse. Fits with two active pairs at $300 \mathrm{GeV}$ and three pairs at $1000 \mathrm{GeV}$ fail completely. 


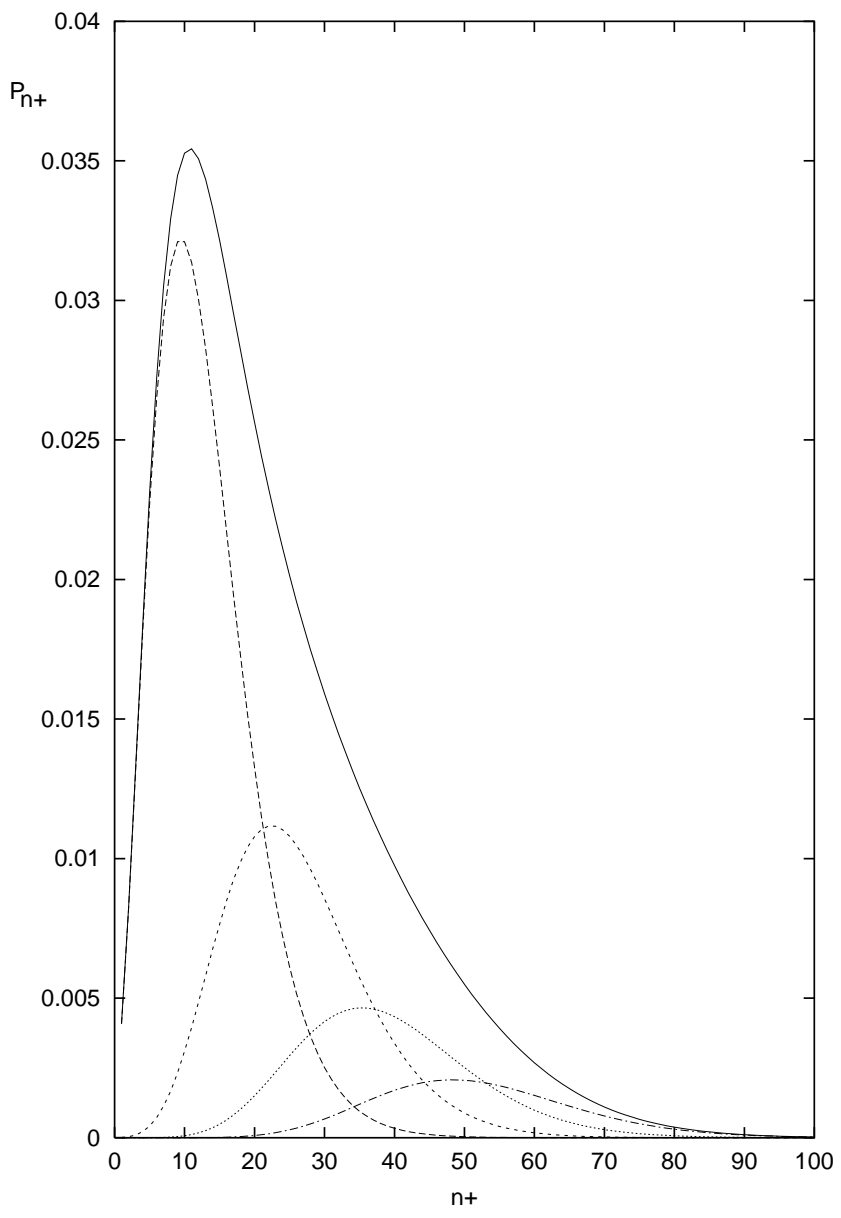

FIG. 4: The decomposition of the fit in Fig. 3 to one, two, three, and four parton-parton collisions.

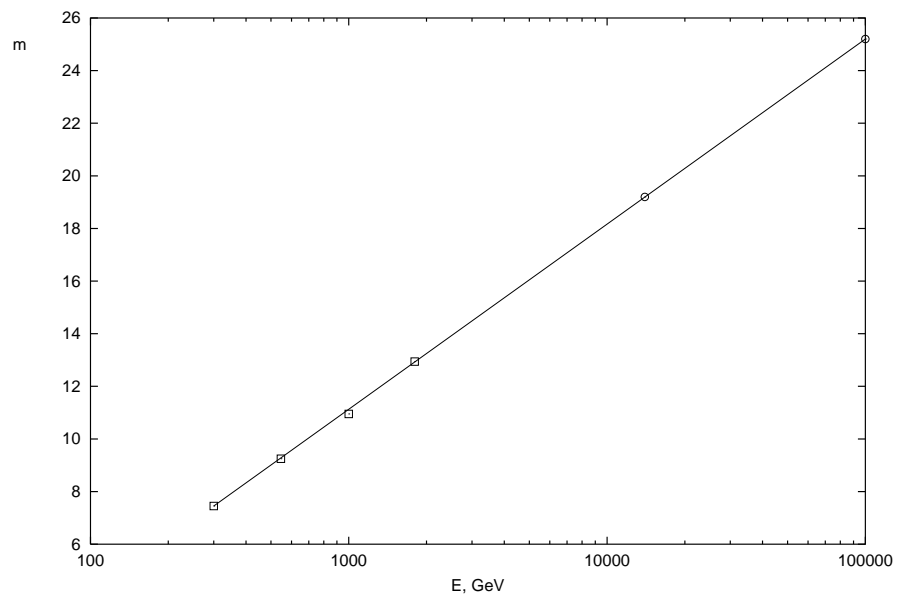

FIG. 5: The energy dependence of $m$ (squares) and its linear extrapolation (circles at 14 and $100 \mathrm{TeV}$ ).

FIG. 6: The values of $k$ as calculated with $w_{j}$ satisfying the relation (8) (squares).
Hence, we have to conclude that this effect results from some dynamics of the hadron interactions that is not understood yet and should be incorporated in the model. The preliminary explanation of this effect could be that at the threshold of new pair formation the previous active pairs produce more squeezed multiplicity distributions due to the smaller phase-space room available for them because of the newcomer. Therefore, the single pair dispersion decreases and the $k$ values increase. This would imply that thresholds are marked not only by the change of $w_{j}$ shown in Table $\llbracket$ but also by the variation of the parameter $k$.

The threshold effects become less important at higher energies. We assume that there are five active pairs at 14 $\mathrm{TeV}$ and six at $100 \mathrm{TeV}$. Then we extrapolate to these energies. The parameter $m$ becomes equal to 19.2 at 14 $\mathrm{TeV}$ and 25.2 at $100 \mathrm{TeV}$ if logarithmic dependence is adopted as shown in Fig. 5 by the straight line. The predicted multiplicity distributions are plotted in Fig. 7. We choose two values of $k$ equal to 4.4 (solid line) and 8 (dash-dotted line) for $14 \mathrm{TeV}$. Low multiplicities are suppressed at larger $k$, and the maximum is slightly shifted to higher multiplicities. The shape of the tail is practically unchanged. For $100 \mathrm{TeV}$, we show only the prediction for $k=4.4$ (dashed line) because increase of $k$ leads to the same qualitative effect as for $14 \mathrm{TeV}$. The oscillations of $H_{q}$ still persist at these energies (see Fig. 8). The minima are, however, shifted to $q=6$ at $14 \mathrm{TeV}$ and 7 at $100 \mathrm{TeV}$ as expected.

The fit at $1.8 \mathrm{TeV}$ with an approximation of $w_{j}$ according to the ladder model (13) with a NBD for a binary parton collision is almost as successful as the fit with values of $w_{j}$ given by the IPPI model. However, some difference at $14 \mathrm{TeV}$ between these models is predicted (compare the solid and dotted lines in Fig. 7, both obtained for $k=4.4$ ). This difference becomes more pronounced at $100 \mathrm{TeV}$. To keep the same mean multiplicity in both models at the same energy, we have chosen different values of $m$ as dictated by Eq. (11) and the $w_{j}$ values shown in Table【 namely, their ratios are $m_{I P P I} / m_{\text {lad }}=0.988$, $1.039,1.123,1.228$ for $j_{\max }=3,4,5,6$, respectively. This shows that the maximum of the distribution moves to smaller multiplicities and its width becomes larger in the ladder model compared to the IPPI model with energy increase.

Certainly, one should not overestimate the success of the IPPI model in its present initial state. It has been applied just to multiplicity distributions. For more detailed properties, say, rapidity distributions, one would need a model for the corresponding features of the one-pair process. Moreover, the screening effect (often described by the triple Pomeron vertex) will probably become more important at higher energies. All these features are implemented in some way in the well known Monte Carlo programs PYTHIA [27], HERWIG 28] and DPM-QGSM 11, 12]. However, for the last one, the multiplicity distribution for a single ladder is given by the Poisson distribution of emission centers (resonances) convoluted with 


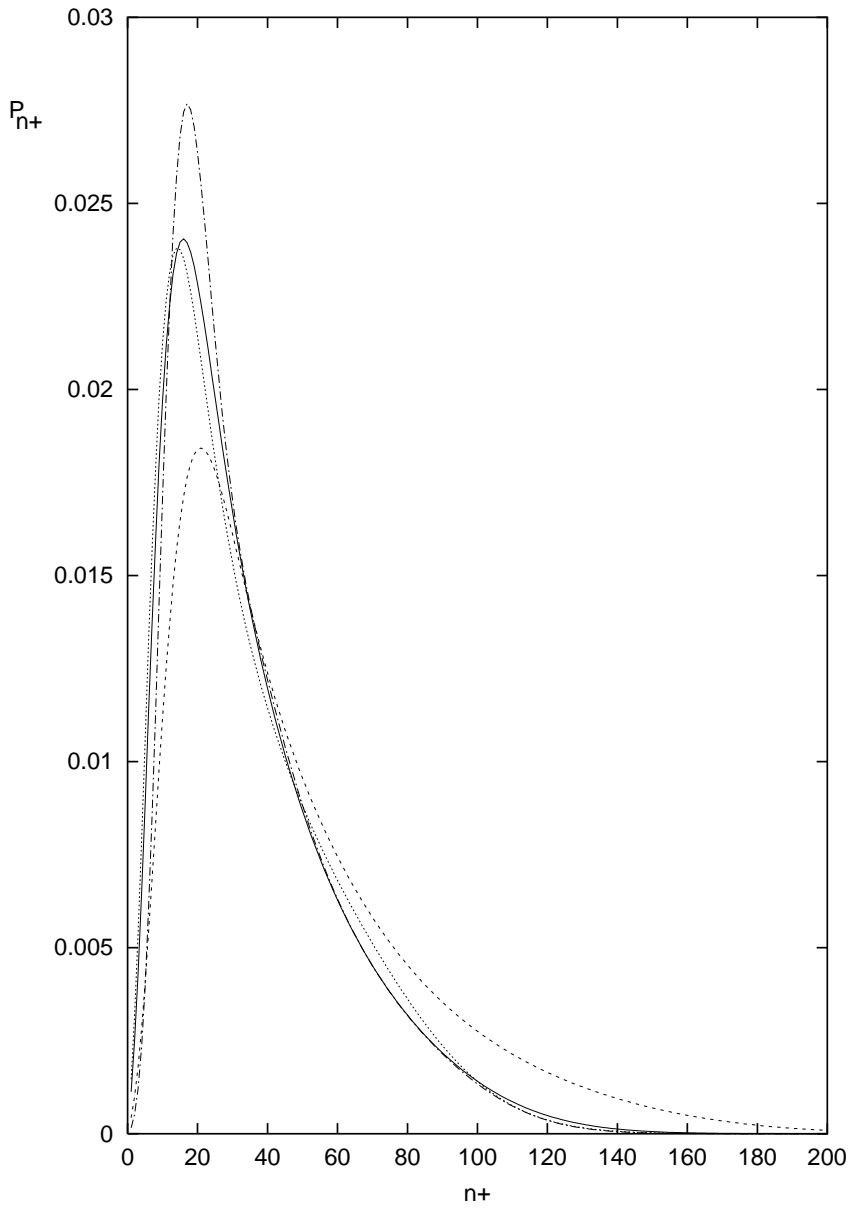

FIG. 7: The same-charge multiplicity distributions at $14 \mathrm{TeV}$ and $100 \mathrm{TeV}$ obtained by extrapolation of parameters $m$ and $k$ with five active pairs at $14 \mathrm{TeV}$ and six at $100 \mathrm{TeV}$ (for the IPPI model: solid line, $14 \mathrm{TeV}, k=4.4$; dash-dotted line $14 \mathrm{TeV}, k=8$; dashed line $100 \mathrm{TeV}, k=4.4$; for the ladder model: dotted line $14 \mathrm{TeV}, k=4.4)$.

their decay properties, and the probabilities $w_{j}$ contain several adjustable parameters. It differs from the IPPI model. The present approach proposes more economic way with a smaller number of such parameters. Concerning the further development of event generator codes, it is tempting to incorporate there the above approach with a negative binomial distribution of particles created by a single parton pair, and confront the results with a wider set of experimental data. This has not been done yet for the IPPI model, and we intend to work on it later to learn how it influences other characteristics.

It would also be interesting to see whether this model is valid for AA collisions as well whether the collective effects (saturation?) prevent its application there. This work is in progress now.

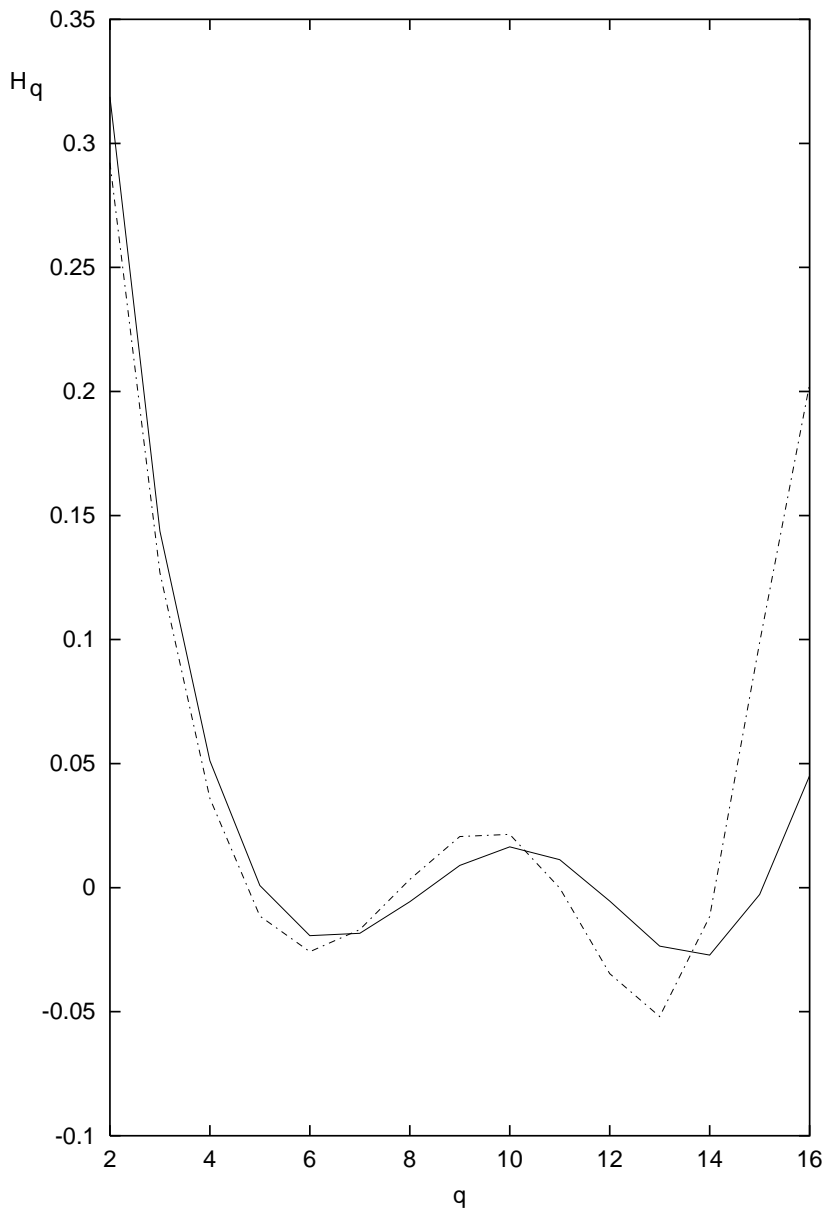

FIG. 8: The behavior of $H_{q}$ predicted at $14 \mathrm{TeV}(k=4.4$, solid line; $k=8$, dash-dotted line).

\section{ARE $e^{+} e^{-}$AND $p \bar{p}$ SIMILAR?}

This question was raised by a recent statement of the PHOBOS Collaboration [29] that the energy behavior of mean multiplicities in all processes is similar. It was concluded that the dynamics of all hadronic processes is the same. In addition to our general belief in QCD, we cannot claim that other characteristics of multiple production processes initiated by different partners coincide.

At first sight, QCD fits of multiplicity distributions in $e^{+} e^{-}$collisions and IPPI model fits of $p \bar{p}$ collisions are completely unrelated and cannot be compared. There is, however, one definite QCD prediction that allows us to ask the question whether QCD and the IPPI model are compatible. This is the asymptotic behavior of $H_{q}$ moments in QCD. They should behave [17] as $H_{q}^{a s}=1 / q^{2}$. One can also determine the asymptotics of $H_{q}^{q}$ moments in the IPPI model and compare both approaches. The asymptotical values of the probabilities $w_{j}(8)$ and their moments $M_{r}$ (10) for $r \leq 5$ are as follows:

$$
\begin{array}{r}
w_{j}=0.5^{j}, M_{1}=2, M_{2}=6, M_{3}=26, \\
M_{4}=150, M_{5}=1082 .
\end{array}
$$


Inserting them in the expressions for $\kappa_{q}$ and $f_{q}$ given in the Appendix, one can evaluate the asymptotic behavior of the $H_{q}$ moments in the IPPI model at any parameter $k$. All asymptotic $H_{q}$ are decreasing functions of $k$. Their minimum values are reached at $k \rightarrow \infty$, i.e., for a convolution of Poisson distributions. They are given by the ratio of the coefficients in front of the leading $k^{q}$ terms in $\kappa_{q}$ and $f_{q}$ (see the Appendix) and are equal to

$$
H_{2}^{(P)}=\frac{1}{3}, H_{3}^{(P)}=\frac{3}{13}, H_{4}^{(P)}=\frac{13}{75}, H_{5}^{(P)}=\frac{75}{541} .
$$

These values are noticeably larger than QCD predictions of $1 / q^{2}$. Since they are even larger for any finite parameter $k$, we have to state that QCD and the IPPI model have different asymptotics. In other words, this implies that Eq. (22), considered as an equation for $f_{q}$ with $H_{q}=1 / q^{2}$ inserted in it, does not have a solution with asymptotical values of $M_{r}$ [Eq. (25)] in the IPPI model.

It is an open question whether other asymptotic relations for $w_{j}$ different from Eq. (8) can be found which would lead to the same behavior of $H_{q}$ moments in $p \bar{p}$ and $e^{+} e^{-}$collisions, i.e., if a solution of Eq. (22) can be found for some values of $M_{r}$ different from those given by Eq. (25). Only then one can hope to declare an analogy between these processes.

Moreover, it has been found from experimental data 18, 30] that the amplitudes of oscillations of $H_{q}$ moments increase for more composite colliding particles. The anomalous fractal dimensions also differ [16] becoming smaller for $A A$ compared with $p \bar{p}$ and even more with $e^{+} e^{-}$. Thus, there is no direct similarity of $e^{+} e^{-}$and $p \bar{p}-$ collisions.

\section{CONCLUSIONS}

To conclude, a model of independent pair parton interactions has been proposed. It is assumed that hadronic interactions proceed through independent parton-parton collisions and each of the binary collisions gives rise to a negative binomial distribution of secondary particles with the same parameters $m$ and $k$. The resulting distribution is described by a weighted sum of NBDs whose parameters are equal to the single collision values $m$ and $k$ multiplied by the number of pairs. Thus no new adjustable parameters appear. Multiple binary parton collisions are assumed to become more important as energy increases. A comparison with experimental data at 300, 546, 1000, and $1800 \mathrm{GeV}$ has shown good agreement. Predictions for the CERN Large Hadron Collider and higher energies are presented. It is demonstrated that asymptotic QCD predictions for $e^{+} e^{-}$multiplicity distributions differ from the asymptotic results of the IPPI model for $p \bar{p}$ processes. Further work on Monte Carlo implementation of this model is in progress.

\section{Acknowledgments}

We are grateful to S. Hegyi for correspondence. This work has been supported in part by the RFBR grants 0202-16779, 04-02-16445-a, and NSH-1936.2003.2. V.N. is also grateful to the Russian Science Support Foundation.

\section{APPENDIX A}

The functions $f_{q}(k)$ and $\kappa_{q}(k)$ are $q$ th order polynomials of $k$ with coefficients determined by the moments $M_{r}$ with $r \leq q$. Their expressions for $q \leq 5$ are as follows:

$$
\begin{array}{r}
f_{1}=\kappa_{1}=M_{1} k, \quad f_{2}=M_{2} k^{2}+M_{1} k, \quad \kappa_{2}=\left(M_{2}-M_{1}^{2}\right) k^{2}+M_{1} k, \\
f_{3}=M_{3} k^{3}+3 M_{2} k^{2}+2 M_{1} k, \quad \kappa_{3}=\left(M_{3}-3 M_{1} M_{2}+2 M_{1}^{3}\right) k^{3}+3\left(M_{2}-M_{1}^{2}\right) k^{2}+2 M_{1} k, \\
f_{4}=M_{4} k^{4}+6 M_{3} k^{3}+11 M_{2} k^{2}+6 M_{1} k, \\
\kappa_{4}=\left(M_{4}-4 M_{1} M_{3}+12 M_{1}^{2} M_{2}-3 M_{2}^{2}-6 M_{1}^{4}\right) k^{4}+6\left(M_{3}-3 M_{1} M_{2}+2 M_{1}^{3}\right) k^{3}+11\left(M_{2}-M_{1}^{2}\right) k^{2}+6 M_{1} k, \\
f_{5}=M_{5} k^{5}+10 M_{4} k^{4}+35 M_{3} k^{3}+50 M_{2} k^{2}+24 M_{1} k, \\
\kappa_{5}=\left(M_{5}-5 M_{1} M_{4}+20 M_{1}^{2} M_{3}-60 M_{1}^{3} M_{2}+30 M_{1} M_{2}^{2}-10 M_{2} M_{3}+24 M_{1}^{5}\right) k^{5}+10\left(M_{4}-3 M_{2}^{2}-4 M_{1} M_{3}\right. \\
\left.+12 M_{1}^{2} M_{2}-6 M_{1}^{4}\right) k^{4}+35\left(M_{3}-3 M_{1} M_{2}+2 M_{1}^{3}\right) k^{3}+50\left(M_{2}-M_{1}^{2}\right) k^{2}+24 M_{1} k .
\end{array}
$$

The ratio of the coefficients in front of the leading $k^{q}$ terms in $\kappa_{q}$ and $f_{q}$ gives $H_{q}$ for the Poisson distribution. Thus, in general, $H_{q}$ differs from 0 for a multicomponent Poisson distribution.
The case of one active pair corresponds to $M_{r} \equiv 1$, and the ordinary formula of NBD is restored: $f_{q}=\Gamma(k+$ q) $/ \Gamma(k)$. 
To demonstrate the accuracy of the $w_{j}$ values shown in Table凹for the IPPI model, we present here their more accurate values and moments for four active parton pairs:

$w_{1}=0.51879, w_{2}=0.26914, w_{3}=0.13963$,

$w_{4}=0.07244, M_{1}=1.76571, M_{2}=4.01103$,

$$
M_{3}=11.0779, M_{4}=34.6791, M_{5}=117.238 \text {. }
$$

These values of $w_{j}$ are larger and those of $M_{r}$ are smaller than the asymptotic ones shown in Eq. (25).
[1] I.M. Dremin and D.S. Chernavsky, ZhETF 38, 229 (1960).

[2] D. Amati, S. Fubini, A. Stanghellini and A. Tonin, Nuovo Cim. 26, 896 (1962).

[3] V.N. Akimov, D.S. Chernavsky, I.M. Dremin and I.I. Royzen, Nucl. Phys. B 14, 285 (1969).

[4] I.M. Dremin and A.M. Dunaevsky, Phys. Rep. 18, 159 (1975).

[5] A. Giovannini and L. Van Hove, Z. Phys. C 30, 391 (1986).

[6] A. Giovannini, Nuovo Cim. A 10, 713 (1972).

[7] P.V. Landshoff and J.C. Polkinghorne, Phys. Rev. D 18, 3344 (1978).

[8] B. Humpert, Phys. Lett. B 131461 (1983).

[9] E735 Collaboration, T. Alexopoulos et al., Phys. Lett. B 435, 453 (1998).

[10] W.D. Walker, Phys. Rev. D (2004) (to be published).

[11] A. Capella, U. Sukhatme, C.I. Tan and J. Tran Thanh Van, Phys. Lett. B 81, 68 (1979).

[12] A.B. Kaidalov and K.A. Ter-Martirosyan, Phys. Lett. B 117, 247 (1982); Sov. J. Nucl. Phys. 40, 135 (1984).

[13] S.G. Matinyan and W.D. Walker, Phys. Rev. D 59, 034022 (1999).

[14] T.K. Gaisser and F. Halzen, Phys. Rev. Lett. 54, 1754 (1985).

[15] A. Giovannini and R. Ugoccioni, Phys. Rev. D 59, 094020 (1999); 68, 034009 (2003).

[16] E.A. De Wolf, I.M. Dremin and W. Kittel, Phys. Rep. 270, 1 (1996).
[17] I.M. Dremin, Phys. Lett. B 313, 209 (1993).

[18] I.M. Dremin and J.W. Gary, Phys. Rep. 349, 301 (2001).

[19] I.M. Dremin and V.A. Nechitailo, Mod. Phys. Lett. A 9, 1471 (1994); JETP Lett. 58, 881 (1993).

[20] I.M. Dremin, V. Arena, G. Boca et al., Phys. Lett. B 336, 119 (1994).

[21] SLD Collaboration, K. Abe et al., Phys. Lett. B 371, 149 (1996).

[22] L3 Collaboration, P. Achard et al., Phys. Lett. B 577, 109 (2003).

[23] E735 Collaboration, F. Turkot et al., Nucl. Phys. A 525, 165 (1991).

[24] UA5 Collaboration, G. Arnison et al., Phys. Lett. B 118, 167 (1982).

[25] Yu.L. Dokshitzer, Phys. Lett. B 305, 295 (1993).

[26] S. Hegyi, Phys. Lett. B 387, 642 (1996); B 417, 186 (1998).

[27] M. Bertini, L. Lönnblad and T. Sjöstrand, Comput. Phys. Commun. 134, 365 (2001); see also http://www.thep.lu.se/ torbjorn/Pythia.html.

[28] HERWIG 6.5, G. Corcella, I.G. Knowles, G. Marchesini, S. Moretti, K. Odagiri, P. Richardson, M.H. Seymour and B.R. Webber, JHEP 0101, 010 (2001); see also http://hepwww.rl.ac.uk/theory/seymour/herwig/

[29] PHOBOS Collaboration, B.B. Back et al., nucl-ex/0301017

[30] I.M. Dremin, V.A. Nechitailo, M. Biyajima and N. Suzuki, Phys. Lett. B 403, 149 (1997). 


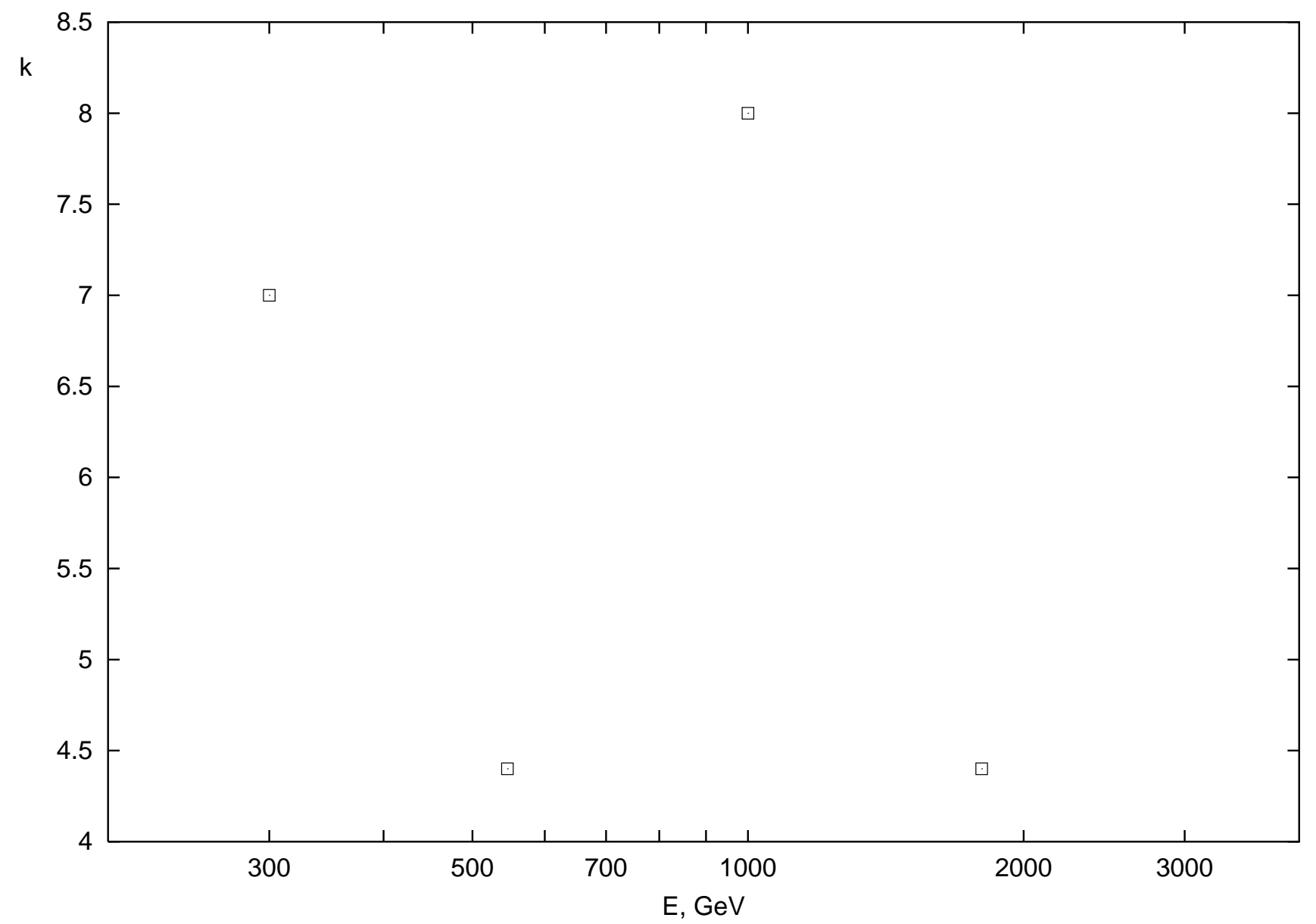

